\title{
Herramientas TIC en la enseñanza online de competencias complementarias de redes y telecomunicaciones en los Institutos Superiores Tecnológicos de la provincia de Tungurahua.
}

\section{TIC tools in online teaching of complementary competences of networks and telecommunications in the Superior Institutes of Tungurahua.}

Santiago Hernán Tisalema Tasigchana. ${ }^{1}$, Christian Omar Borja Guevara. ${ }^{2}, \&$ William Arturo Godoy Arce. $^{3}$

\section{Resumen DOI: https://doi.org/10.33262/concienciadigital.v3i4.1448}

This research project is essentially a brief analysis of the use and advantages of Information and Communication Technologies - ICT in the Higher Technological Institutes of Ambato, Tungurahua province, therefore the article is focused on studying the case of the Technology Career Superior in Networks and Telecommunications of the Higher Technological Institute "Luis A. Martínez"; for this, a preliminary diagnosis of the competences that students have in ICT was planned and executed, then the most versatile, simple and efficient computer tools that can be used strategically in the teaching and learning of complementary competencies of the career are highlighted. It was worked fundamentally under a mixed approach, and a consequent empirical and mixed methodology, same that at the beginning was clearly documentary and at the end a quasiexperimental phase was applied with a significant and representative sample of 108 randomly chosen students, with this it was possible to compare the effects and acceptance of ICT tools in basic subjects and specialty subjects through questionnaires applied to active students in the period 2019-II, that is, from November 2019 to April 2020, and finally with the information collected, a summary is prepared that suggests some websites and simulators available to all members of the institute's educational community.

Keywords Tic, Networks, Telecommunications, Skills.

\section{Resumen}

El presente proyecto de investigación es esencialmente un breve análisis del uso y ventajas de las Tecnologías de la Información y Comunicación - TIC en los Institutos

\footnotetext{
1 Instituto Superior Tecnológico Luis A. Martínez, Ambato, Ecuador, santiagohernantisalema@gmail.com, https://orcid.org/0000-0002-4851-0996.

${ }^{2}$ Instituto Superior Tecnológico Luis A. Martínez, Ambato, Ecuador, barcecobg@ hotmail.com

${ }^{3}$ Instituto Superior Tecnológico Luis A. Martínez, Ambato, Ecuador, williamgodoyarce@gmail.com
} 
Superiores Tecnológicos de Ambato provincia de Tungurahua, por ello el artículo está enfocado en estudiar el caso de la Carrera de Tecnología Superior en Redes y Telecomunicaciones del Instituto Superior Tecnológico "Luis A. Martínez"; para ello se planificó y ejecutó un diagnóstico preliminar sobre las competencias que poseen los estudiantes en las TIC, luego se destaca las más versátiles, sencillas y eficientes herramientas informáticas que se pueden utilizar estratégicamente en la enseñanza y aprendizaje de competencias complementarias de la carrera. Se trabajó fundamentalmente bajo un enfoque mixto, y una consecuente metodología empírica y mixta, misma que al principio fue netamente documental y al final se aplicó una fase cuasi experimental con una muestra significativa y representativa de 108 estudiantes escogidos aleatoriamente, con ello se logró comparar los efectos y aceptación de herramientas Tic en las asignaturas básicas y en asignaturas de especialidad mediante cuestionarios aplicados a estudiantes activos en el período 2019-II , es decir desde noviembre 2019 hasta abril 2020, y finalmente con la información recopilada se elabora un resumen que sugiere algunos sitios web y simuladores al alcance de todos los miembros de la comunidad educativa del instituto.

Palabras clave: Tic, Redes, Telecomunicaciones, Competencia

\section{Introducción.}

Aproximadamente hace una década, los órganos rectores de la educación superior en el Ecuador impulsaron cambios que implican una mejora considerable de los índices de calidad mediante reformas legales que orientan a la diversificación de las funciones sustantivas mencionadas por (Consejo de Educación Superior, 2019, pág. 4), en el Reglamento de Régimen Académico vigente, y en concordancia con el artículo 117 de la (LOES, 2010), como son :

a) La docencia, que implica la construcción de conocimientos y desarrollo de capacidades y habilidades, como resultado de la interacción entre profesores y estudiantes en experiencias de enseñanza-aprendizaje (Consejo de Educación Superior, 2019).

b) La vinculación con la sociedad, que consiste en generar capacidades e intercambio de conocimientos en el estudiante, en función de los dominios académicos de las instituciones de educación superior, con la finalidad de aportar con soluciones a las necesidades y desafíos de su entorno (LOES, 2010), y,

c) La investigación e innovación tecnológica, que según (Consejo de Educación Superior, 2019), potencia los conocimientos y saberes científicos, ancestrales e interculturales.

Adicionalmente implicó la aplicación de nuevos paradigmas, modelos educativos y metodologías. Uno de los cambios importantes según (LOES, 2010), es que se distinguió al docente como docente - investigador, buscando incentivar a la comunidad educativa a generar conocimiento en función del cambio de la matriz productiva del país. Dichos 
cambios incidieron en todas las instituciones de educación superior del país, tales como: Universidades y escuelas politécnicas, institutos y conservatorios superiores, etc.

En la búsqueda de mejorar la capacidad productiva del país aproximadamente en 2014, se decidió vincular oficialmente a la educación superior a los institutos superiores técnicos y tecnológicos del Ecuador, con rediseños de carreras enfocadas a mejorar las capacidades del personal técnico y promoviendo de esta forma, la mano de obra calificada en la utilización de equipos, máquinas - herramientas y nuevas tecnologías de la información tanto en el campo comercial, industrial y otras áreas actuales del conocimiento.

Como una alternativa de respuesta a las necesidades del sector productivo se crean las carreras tecnológicas en el campo de las TICS, y entre ellas se destaca la Carrera de Tecnología Superior en Redes y Telecomunicaciones , misma que desde su aprobación (CES, 2017) esta en vigencia con el objeto de proporcionar competencias técnicas para operar y manipular equipos de interconexión de redes de datos y proporcionar soluciones de comunicaciones electrónicas mediante la configuración e interacción con equipos en su mayoría con tecnologías digitales con proyección a redes con tecnología óptica: G-PON.

Según el rediseño aprobado por (CES, 2017), la carrera de Redes y Telecomunicaciones, está constituida básicamente por cuatro campos:

a. Fundamentos teóricos: Matemática, Algebra Booleana, Geometría Analítica, entre otras.

b. Adaptación e innovación tecnológica, entre las asignaturas más importantes tenemos: Electricidad y Magnetismo, Electrónica Básica, Fundamentos de Redes y Telecomunicaciones, Utilitarios de Redes, Sistemas de Comunicación, Enlaces de Comunicación Inalámbricas, Seguridad de Redes.

c. Comunicación y lenguajes: Expresión Oral y escrita, Technical English , entre otras.

d. Integración de saberes, contextos y culturas: Realidad Socioeconómica y Política del Ecuador, Ecología y Educación Ambiental.

Del listado anterior existen algunas asignaturas en las que se puede insertar conocimientos de mejor forma mediante herramientas Tic, complementando las competencias planificadas inicialmente en la malla, como es el caso de Electrónica Básica en la que se puede afianzar conocimientos mediante simuladores de circuitos electrónicos como Proteus o Livewire en donde se puede estudiar el comportamiento de elementos o se puede diseñar los esquemas impresos.

\section{Realidad de las TICS en la Educación Superior del Ecuador}

Aunque las autoridades de educación superior han propuesto grandes cambios mediante procesos de diagnóstico y evaluación institucional llevadas a cabo desde el 2014 con la finalidad de cumplir con estándares de calidad que aparentemente benefician a la comunidad 
educativa, se puede decir que aún se observan bajos niveles de eficacia, eficiencia y pertinencia, pues la calidad alcanzada por las IES y diversos sistemas educativos superiores , no cumplen totalmente con las expectativas y nuevas exigencias de la sociedad, respecto a la inclusión de las Tecnologías de Información y Comunicación (Castellanos, 2015).

La prueba de ello, son los resultados de las primeras evaluaciones aplicadas por el CEAACES , desde 2009 hasta aproximadamente el 2012, donde se registraron muy pocas entidades de educación superior como la Escuela Superior Politécnica del Ejército (ESPE) o la Universidad San Francisco de Quito (USFQ) que fueron declaradas como acreditas con categoría A, así mismo la mayoría de Universidades del país fueron designadas con categoría B o categoría C , y finalmente un gran número de entidades que no lograron acreditarse y por lo tanto el CES dispuso su cierre debido a sus bajos índices de calidad especialmente en el criterio de infraestructura, tanto física como tecnológica.

Por otro lado, la realidad de la infraestructura tecnológica de los institutos superiores ecuatorianos ( ISTT) también es preocupante, ya que hasta el 2020 no se ha cumplido aquella idea de potenciar a la educación tecnológica y la prueba de ello está en que con una población de más de un centenar de institutos funcionando antes del 2014, solamente se registran alrededor de cuarenta ISTT acreditados, y el problema radica en que la mayoría está considerado condicionado o fuertemente condicionado, todo ello debido precisamente a los bajos niveles de calidad de la infraestructura.

Por ello se puede decir que la dotación de infraestructura tecnológica y soporte TIC es muy importante para que los estudiantes desarrollen sus competencias complementarias en función de consumar el perfil de egreso de su carrera, especialmente en áreas tecnológicas o productivas.

Las Tecnologías de la Información y Comunicación, en la actualidad se han convertido en un auténtico canal de comunicación entre los diferentes entes de la sociedad estableciendo importantes relaciones hacia el desarrollo conjunto, y, por ende, implica un tratamiento oportuno dentro de los procesos de enseñanza-aprendizaje en las universidades y con mucha más razón en institutos superiores del Ecuador, pero no sólo en el proceso como tal, sino, sobre todo, en su organización y gestión (Ruiz, Martínez, \& Sánchez, 2016).

Por lo antes mencionado en la presente investigación se propone indagar sobre los tipos de herramientas TIC que se pueden utilizar, con la finalidad de optimizar los recursos y la inadecuada infraestructura tecnológica que se dispone en los institutos superiores tecnológicos del Ecuador partiendo de un diagnóstico breve sobre la aplicación de diferentes herramientas TIC y sus probables ventajas en el aprendizaje de temáticas complementarias de las carreras de tecnología superior en Redes y Telecomunicaciones.

En el área de simulación y configuración de redes se suele utilizar Packet Tracer versión estudiantil de propiedad de la multinacional CISCO, sin embargo existen otras herramientas 
que permiten complementar el fundamento teórico y permiten optimizar recursos mediante modelados por computadora, ya sea en línea o de forma desatendida, como es el caso de la herramienta Geogebra, que es un software gratuito muy utilizado en las áreas básicas.

\section{Generalidades de las Tecnologías de la Información y Comunicación}

Se estima que las TIC prácticamente nacen al mismo tiempo que las computadoras, las redes o las telecomunicaciones, existen varias relaciones conceptuales acerca de su significado, por mencionar algunas tenemos:

Para (García-Valcárcel, 2001) las TIC, son todos aquellos medios que surgen a raíz del desarrollo de la microelectrónica, fundamentalmente los sistemas de video, informática y telecomunicaciones.

Las tecnologías de información y comunicación, son aquellos dispositivos, herramientas, equipos y componentes electrónicos, capaces de manipular información, que en conjunto soportan el desarrollo y crecimiento económico de cualquier organización (Thompson \& Strickland, 2004).

Según (Tello, 2011, pág. 10), TIC : “el conjunto de herramientas, soportes y canales para el proceso y acceso a la información, que forman nuevos modelos de expresión, nuevas formas de acceso y recreación cultural"

En concordancia, a lo antes mencionado, las TIC, son todos aquellos medios tecnológicos relacionados a la electrónica y a las telecomunicaciones que permiten transmitir información de una manera oportuna y sencilla acorde a los recursos y necesidades de la sociedad.

En adición (Gallardo \& Buleje, 2010) manifiesta que los principales factores que influyen en el uso de las TIC en el ámbito académico son: el acceso a diferentes recursos, la calidad de software y hardware, ductilidad o sencillez de uso, el apoyo y la solidaridad de las instituciones para usar estas herramientas en el currículo, las políticas nacionales y locales son un compromiso con la superación profesional, y la capacitación formal

Por citar lagunas herramientas TIC en la educación superior tecnológica tenemos:

- Plataformas virtuales

- Videoconferencias

- Simuladores online

- Simuladores

- Páginas web

- Calculadoras online

- Aplicaciones móviles 


\section{Metodología.}

Para el presente trabajo, la metodología se orienta en las siguientes fases:

a) Análisis de la información teórica disponible en diferentes fuentes bibliográficas.

b) Diagnóstico de la utilización de herramientas TIC en la mayoría de asignaturas de la malla de la carrera de redes y telecomunicaciones.

c) Recolección, tratamiento y resumen de herramientas TIC como alternativa para mejorar competencias académicas.

d) Valoración de resultados de la aplicación de TICS en una muestra representativa.

Una vez establecida la metodología a aplicarse, se procedió como sigue:

\section{a) Análisis de la información teórica disponible en diferentes fuentes bibliográficas.}

La investigación inició con la revisión de fuentes bibliográficas primarias que según (Biblioteca de la Universidad de Alcalá, 2020) son: libros, artículos de revistas científicas, documentos e informes oficiales, etc. Para el caso la información se complementa con fuentes secundarias como páginas web relacionadas al tema investigado.

\section{b) Diagnóstico preliminar de la utilización de herramientas TIC en la mayoría de asignaturas de la malla de la carrera de redes y telecomunicaciones.}

Para ejecutar dicho diagnóstico preliminar se escogió aleatoriamente tres asignaturas por cada campo de organización de las asignaturas según la malla de redes y telecomunicaciones (CES, 2017).

Con ello, se diseñó un formulario en línea a modo de cuestionario con la finalidad de obtener la información necesaria que permita diagnosticar el nivel de conocimiento y utilización de las TIC por parte de los docentes y estudiantes del instituto a fines de diciembre del 2019.

Como el presente estudio se enfoca precisamente a competencias complementarias de la carrera de Redes y Telecomunicaciones del Instituto Superior Tecnológico Luis A. Martínez del centro de Ambato, desde noviembre del 2019 hasta Junio del 2020, la población la constituyen los 150 estudiantes que cursan la carrera desde primero a quinto semestre.

Para la elaboración del cuestionario se tomó en cuenta las escalas de Likert, y posteriormente se lo validó a través del método del Alfa de Cronbach, que permite establecer el grado de consistencia de un instrumento de evaluación y determinar el nivel de confianza que tendrá al ser usado (Bojórquez Molina, López Aranda, Hernández Flores, \& Jiménez López, 2013).

Para determinar el tamaño de la muestra se utilizó la fórmula para una población finita (Gallego, 2004), con un nivel de confianza es del $95 \%$ y un margen de error del $5 \%$, como 
resultado del cálculo se obtuvo 108 personas a las que finalmente se les selecciona mediante un muestreo aleatorio simple previo la aplicación de las encuestas.

\section{Cálculo de la muestra preliminar:}

$$
n=\frac{Z^{2} * p * q * N}{\left(e^{2} *(N-1)\right)+Z^{2} * p * q}
$$

Donde se conoce previamente lo siguiente:

Nivel de confianza (95\%): $Z=1,96$

Error Muestral: e $=5 \%$

Población: $\mathrm{N}=150$

Probabilidad de éxito : $p=0,50 \quad(50 \%)$

Probabilidad de fracaso $(1-\mathrm{p})=\mathrm{q}=0,50$

$$
\begin{aligned}
& n=\frac{1,96^{2} * 0,50 * 0,50 * 150}{\left(0,05^{2} *(339-1)\right)+1,96^{2} * 0,5 * 0,5}=108,08 \\
& n \approx 108
\end{aligned}
$$

\section{c) Recolección, tratamiento y resumen de herramientas TIC como alternativa para mejorar competencias académicas.}

En Ecuador aproximadamente en 2012 funcionan alrededor de 250 institutos superiores técnicos y/o tecnológicos, entre públicos y privados, para el 2014 aquella cifra se redujo luego del proceso de evaluación ejecutado por el CEAACES, más tarde en 2018 se contabilizaban cerca de 140 institutos públicos y el resto son particulares, en los que se oferta decenas de carreras de áreas diferentes la mayoría de dichas carreras son de modalidad o carácter presencial, solamente un pequeño porcentaje de carreras funcionan bajo la modalidad dual, y muy pocas carreras son de modalidad semipresencial.

Para el presente trabajo fue imprescindible indagar la cantidad de carreras relacionadas con el campo de las TIC, por lo que se optó por tabular dicha información partiendo de la información que provee la base de datos del SNIESE donde, en la Tungurahua se registran alrededor de 8 carreras tecnológicas relacionadas con el campo de las TIC, específicamente en los institutos: IST Guayaquil , IST Bolívar, IST Pelileo, IST Luis A. Martínez del centro de Ambato, y por lo pronto es el único Instituto en la provincia que posee la carrera de Redes y Telecomunicaciones.

Con la información pertinente acerca de las herramientas TIC se elaboró un listado y posterior resumen de las herramientas TIC, que se sugieren al docente de la mencionada institución de educación superior, para estimular el aprendizaje de algunas asignaturas de la malla de la carrera de Redes y Telecomunicaciones. 
finalmente se hará una prueba de campo que consiste en escoger un grupo de asignaturas, docentes y estudiantes, e insertar en la planificación de clase el aprendizaje autónomo mediante clases online o tutorías online que propongan prácticas online y luego de unos 90 días evaluar mediante encuestas online sobre las ventajas y el nivel de aceptación adquiridos por los estudiantes en estudio.

\section{d) Valoración de resultados de la aplicación de TICS en una muestra selectiva.}

Tomando en cuenta las herramientas sugeridas en la fase anterior, se escogió otra muestra con igual cantidad de estudiantes que la primera fase $(n=108)$ pero con la diferencia de que en esta oportunidad se seleccionó varias asignaturas a fin de completar la muestra, es así que se las selecciona de acuerdo al siguiente criterio:

- Dos asignaturas especialidad y complementarias como: Electrónica básica y Sistemas de Comunicación ( total 50 estudiantes).

- Dos asignaturas de áreas básicas como: Matemática y Algebra Booleana ( total 58 estudiantes )

Se propuso aplicar un cuasi experimento desde enero hasta abril 2020 y luego se aplicó un nuevo cuestionario con el que se pretende determinar el nivel de aceptación y satisfacción de conocimientos adquiridos mediante herramientas TIC, y para ello se ha insertado el uso de herramientas web y simuladores en las asignaturas seleccionadas excepto en Sistemas de Comunicación.

\section{Resultados y Discusión}

\section{Resultados del diagnóstico preliminar del uso de las TIC en estudiantes de la carrera de Redes y telecomunicaciones}

El cuestionario fue validado previamente con éxito, ya que se obtuvo como coeficiente de Alfa de Cronbach de 0,905; pues según (George \& Mallery, 2003) se considera excelente acorde al grado de fiabilidad del mismo.

Tabla 1: Grado de fiabilidad mediante alfa de Cronbach

\begin{tabular}{ll}
\hline $\begin{array}{l}\text { Coeficiente } \\
\text { Alfa Cronbach }\end{array}$ & $\begin{array}{l}\text { Grado de } \\
\text { Fiabilidad }\end{array}$ \\
\hline$>\mathbf{0 , 9}$ & Excelente \\
\hline$>\mathbf{0 , 8}$ & Bueno \\
\hline$>\mathbf{0 , 7}$ & Aceptable \\
\hline$>\mathbf{0 , 6}$ & Cuestionable \\
\hline$>\mathbf{0 , 5}$ & Pobre \\
\hline$<\mathbf{0 , 5}$ & Inaceptable \\
\hline
\end{tabular}

Fuente: (George \& Mallery, 2003)

Elaborado por: Autores de la investigación 
Tomando en consideración este grado de fiabilidad se puede aplicar el cuestionario sin ninguna observación al respecto.

\section{Disponibilidad de Medios tecnológicos}

Es importante observar los datos obtenidos en cuanto a la disponibilidad de medios tecnológicos para establecer implícitamente el uso de las TIC en los estudiantes y docentes de la institución, pues se podría decir que la aplicación de herramientas TIC no serán posibles sin los recursos necesarios, ante ello se presentan los siguientes resultados:

Ilustración 1. Disponibilidad de medios tecnológicos en estudiantes de la carrera de Redes y Telecomunicaciones del Instituto Superior Tecnológico "Luis A. Martínez"

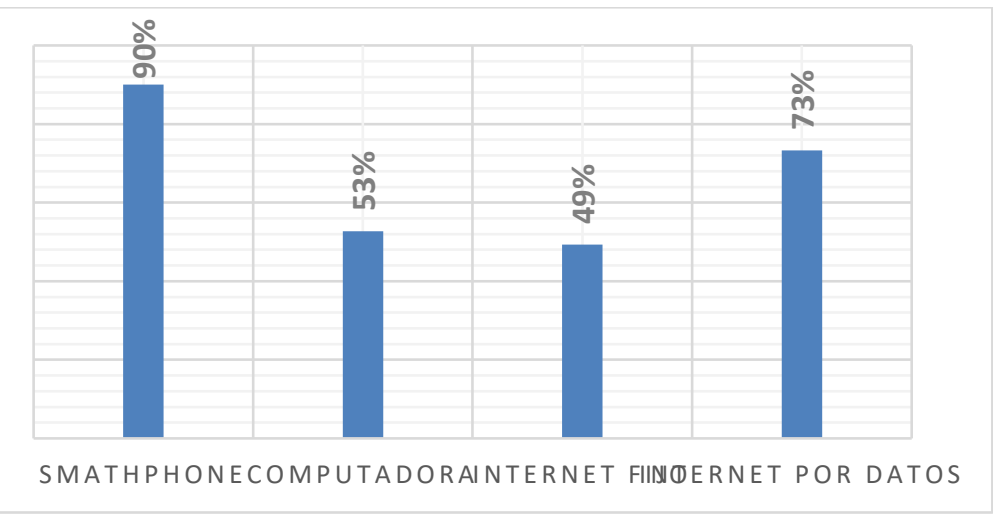

Fuente: Trabajo de campo

Elaborado por: Autores de la investigación

Según se observa la mayoría de estudiantes usan smartphone, sin embargo, solamente la mitad disponían de computador y de internet fijo en casa, siendo esto un resultado nada alentador en función de los requerimientos del uso de las TIC.

Ilustración 2. Uso de herramientas TIC en estudiantes de la carrera de Redes y Telecomunicaciones del Instituto Superior Tecnológico "Luis A. Martínez"

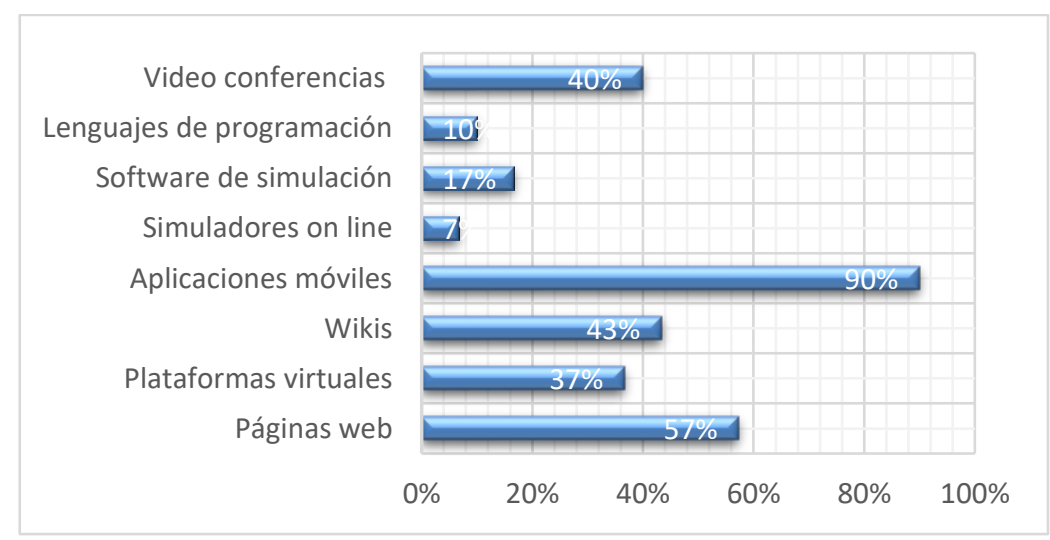

Fuente: Trabajo de campo

Elaborado por: Autores de la investigación

Como se puede observar en la ilustración N. 2 , en diciembre 2019 el uso de TIC en estudiantes de la carrera es bastante deficiente, pues solamente la mitad de los encuestados 
sostiene que sabe utilizar páginas web, solamente la tercera parte tiene experiencia en el uso de plataformas virtuales, y 4 de cada cinco estudiantes no han utilizado simuladores para probar sus circuitos o sus topologías redes LAN.

Ilustración 3. Nivel de conocimiento de herramientas TIC en estudiantes de la carrera de Redes y Telecomunicaciones del Instituto Superior Tecnológico "Luis A. Martínez"

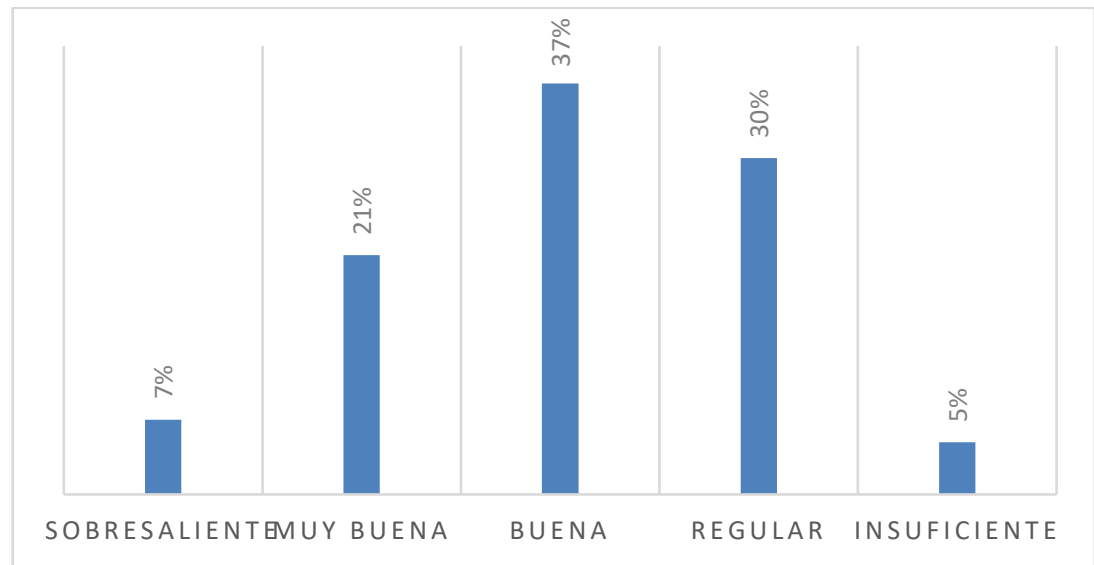

Fuente: Trabajo de campo

Elaborado por: Autores de la investigación

En concordancia de los resultados obtenidos anteriormente se puede manifestar que prácticamente el $65 \%$ de los estudiantes considera que no posee un nivel adecuado en cuanto a utilización de recursos TIC a pesar de ser estudiantes de áreas tecnológicas.

Prácticamente al 2019 se puede manifestar que las dos terceras partes de la muestra en estudio no poseen competencias adecuadas o no se les ha incentivado a trabajar con herramientas de software que complementen su formación técnica -tecnológica

Ilustración 4. Nivel de persepción de estudiantes sobre el uso de TICS en las asignaturas de la carrera de Redes y Telecomunicaciones del Instituto Superior Tecnológico "Luis A. Martínez"

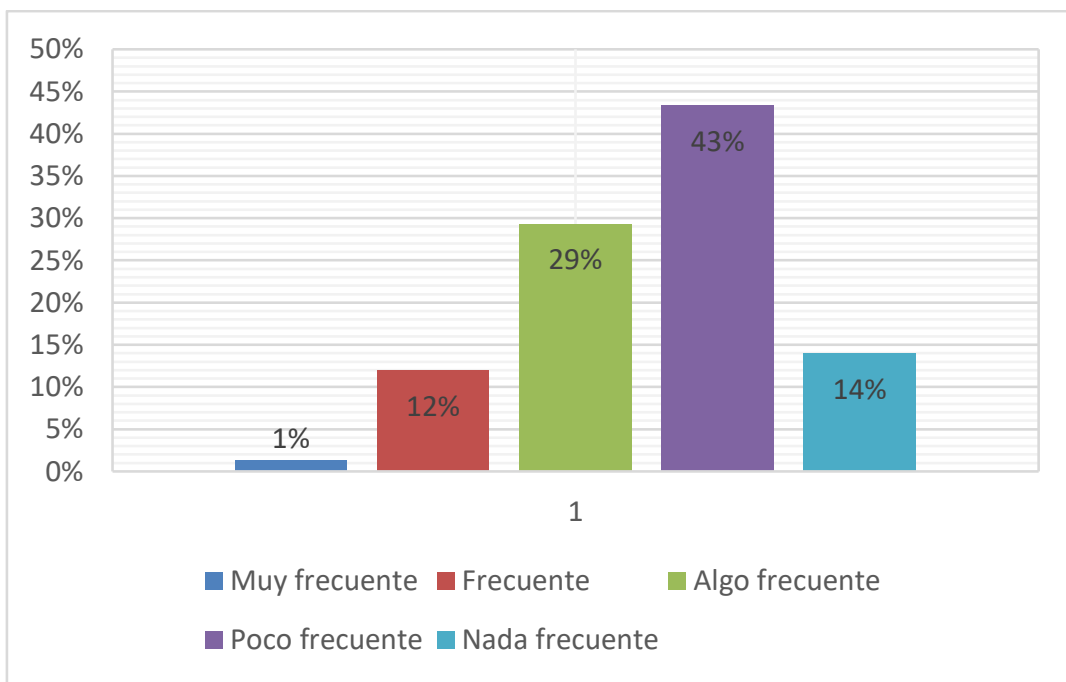

Fuente: Trabajo de campo

Elaborado por: Autores de la investigación 
La mitad de la muestra indica que en promedio los docentes de la institución no implementan con frecuencia el uso de TIC dentro de sus recursos de la clase

\section{Resumen de herramientas TIC que se pueden utilizar como alternativa para mejorar competencias académicas.}

Luego de la etapa de búsqueda de información pertinente se puede decir que en el internet existe una gran diversidad de herramientas digitales dispuestas generalmente en dos versiones, la completa (pagada) y la versión gratuita o estudiantil, mismas que se pueden implementar en diversos campos del conocimiento pues son excelentes recursos TIC, tanto para educación online como presencial; en tal virtud se ha resumido en cinco grandes grupos de herramientas TIC, usadas por lo general por académicos en la educación superior, tales como:

A. Plataformas virtuales: son considerados como grandes sitios web que permiten configuración por niveles jerárquicos con la finalidad de impartir todo tipo de capacitaciones o formación mediante internet sin necesidad de tener conocimientos de programación (aula1, 2020). Por lo general incluyen varios servicios como: avisos, servicios de mensajería, chat, envío de archivos, foros, etc.

Existen tres tipos de plataformas virtuales:

1. Plataformas educativas comerciales: WebCT

2. Plataformas de software libre: Moodle

3. Plataformas de desarrollo propio: plataformas de software libre y código abierto

B. Sitios web con servicios online: son aplicaciones web desarrolladas para permitir al usuario probar, utilizar, calcular o simular ciertas funciones en línea, en este grupo destacan dos tipos:

1. Simuladores online para ciencias exactas, que permiten efectuar cálculos matemáticos, estadísticos , matriciales o trigonométricos y graficas de funciones; como: Geogebra, Wolfram alpha, Simbolab, Mathway.

2. Simuladores online para circuitos eléctricos y electrónicos, como: DCACLAB, Logic Circuit Test, etc. La desventaja es que solamente permiten probar circuitos simples.

C. Software de Simulación offline, en este grupo de herramientas TIC se encuentran una gran variedad de programas ejecutables que permiten la simulación de circuitos electrónicos como Proteus o Livewire, otros que se destacan por la simulación de la conexión y configuración de equipos de redes como Packet tracer u otros programas que permiten simular dispositivos industriales, siendo su principal desventaja el costo de la licencia o su carga computacional alta (programas pesados). 
D. Aplicaciones móviles, disponibles desde un smartphone con servicio de internet, tal como: Mathgraph,

E. Herramientas multimedia, (Appsherramientasmultimediaonlineval, 2016) permiten preparar material didáctico o editar tareas mediante recursos multimedia, tal como: Examview, YouTube, etc.

\section{Resultados de la aplicación de TICS en una muestra selectiva.}

En esta etapa se propone aplicar un cuasi experimento en la muestra selectiva de 108 estudiantes de la carrera e institución mencionada, que consiste en valorar los cambios que se han dado y la percepción de los estudiantes, luego de aplicar a propósito la inserción de herramientas TIC en la enseñanza de los contenidos según lo planificado en el periodo de noviembre 2019 hasta abril del 2020.

Se seleccionó cuatro asignaturas de la carrera y en ellas se aplicó experimentalmente el uso de las siguientes herramientas TIC:

a. Matemática (primer semestre): se sugiere trabajar con Geogebra online y Simbolab, para comprobar las soluciones de los ejercicios propuestos y en el caos del docente explicar visualmente mediante videoconferencia.

b. Algebra Booleana (segundo semestre): se sugiere trabajar con el simulador denominado Livewire (Lucas, 2009) , para probar expresiones booleanas combinadas.

c. Electrónica Básica (segundo semestre): se sugiere trabajar con croco- dile technology o crocodile clips para simular el funcionamiento de los circuitos básicos y comprobar la ley de ohm (FisicaLab, 2020).

En 2020 los datos obtenidos en cuanto a la disponibilidad de medios tecnológicos son las siguientes:

Ilustración 5. Disponibilidad de medios tecnológicos en estudiantes

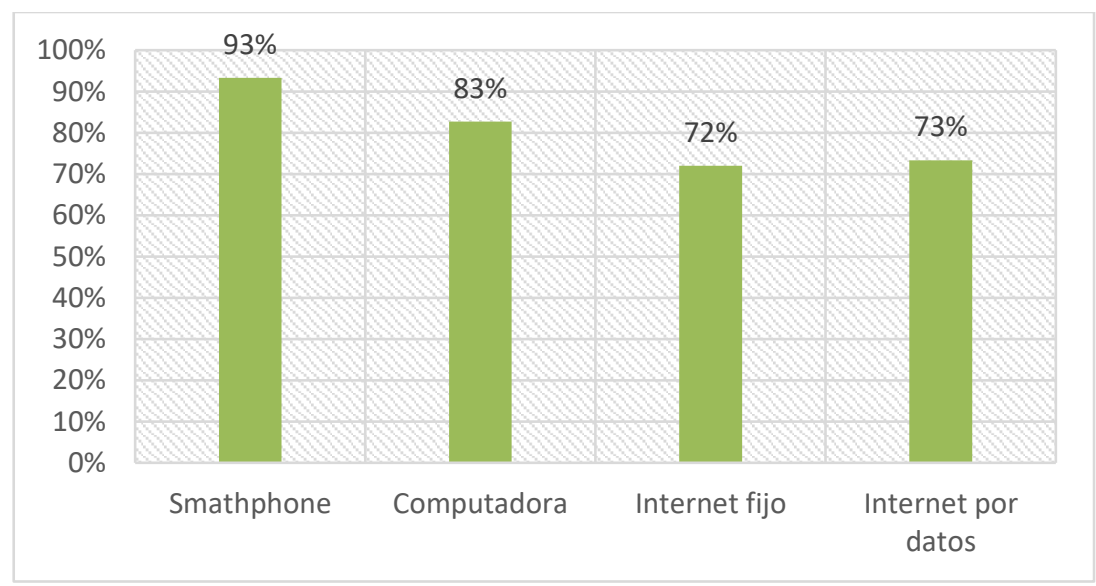

Fuente: Trabajo de campo

Elaborado por: Autores de la investigación 
Según se observa ocho de cada diez estudiantes disponen de computador, y las tres cuartas partes han adquirido internet fijo en casa, siendo esto un resultado aliciente para la aplicación general y uso de las TIC.

Ilustración 6. Uso de herramientas TIC

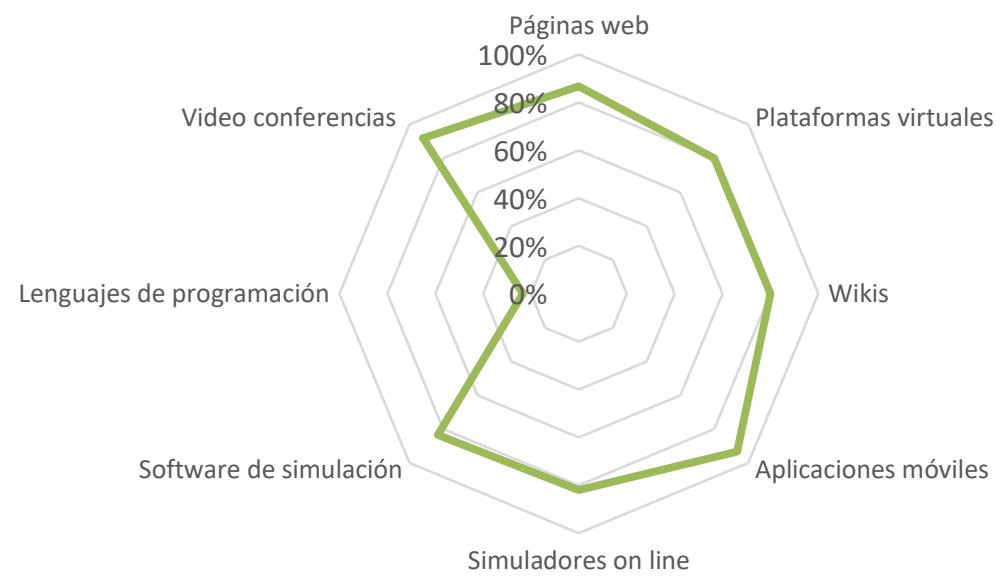

Fuente: Trabajo de campo

Elaborado por: Autores de la investigación

Como se puede observar en la ilustración N.6, a fines de abril 2020, el uso de TIC en estudiantes de la carrera ha cambiado de deficiente a aceptable, sin embargo la mayoría coincide en el desconocimiento de lenguajes de programación, aunque ya tienen experiencia en el uso de plataformas virtuales, y han utilizado simuladores para probar sus circuitos o sus topologías redes LAN.

Ilustración 7. Nivel de conocimiento de herramientas TIC

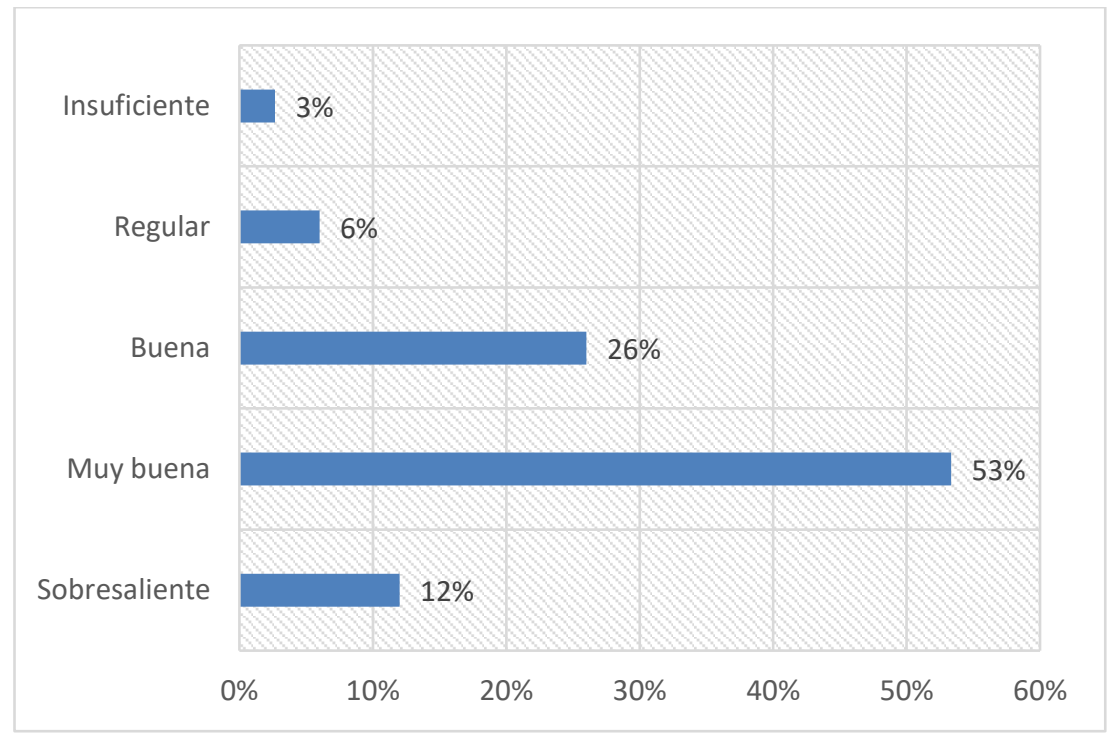

Fuente: Trabajo de campo

Elaborado por: Autores de la investigación 
Como se puede observar en la ilustración 7 , se puede manifestar que prácticamente las tres cuartas partes de los estudiantes han mejorado su nivel de competencia en cuanto a la adecuada utilización de recursos TIC.

Ilustración 8. Nivel de persepción de estudiantes sobre el uso de TIC específicas

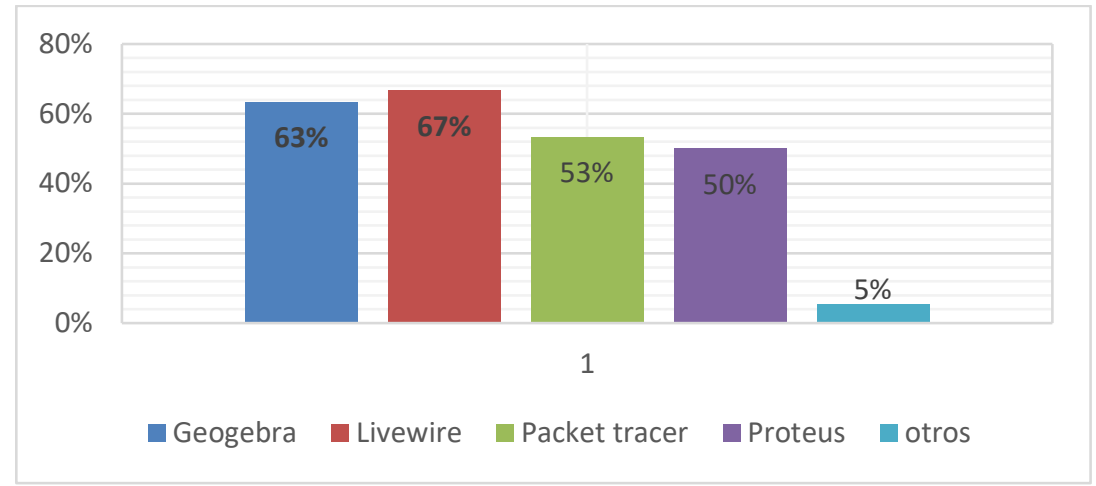

Fuente: Trabajo de campo

Elaborado por: Autores de la investigación

Los resultados obtenidos indican que más de la mitad de los estudiantes de redes y telecomunicaciones han aceptado positivamente la inserción de programas y simuladores, y por tanto ha mejorado sus competencias cognitivas.

\section{Discusión}

Comparando los resultados obtenidos antes y después de realizar un cuasi experimento aplicado a la muestra de 108 estudiantes de la carrera de redes y telecomunicaciones, se puede decir que la aplicación de herramientas TIC implican la adquisición de equipos tecnológicos e internet lo que evidentemente a mejorado.

A pesar de la considerable evolución en el plano tecnológico, aun no se ha llegado a niveles óptimos y la prueba está en que se ha incrementado en aproximadamente un 30\% el uso las herramientas TIC como es el caso del uso de las plataformas virtuales, simuladores y videoconferencias, sin embargo hay que comprender que la mejoría no se debe a políticas del estado, sino más bien se debe a varios factores externos como la inserción de la modalidad online debido al confinamiento obligatorio como consecuencia de la pandemia lo que a su vez implicó una creciente necesidad de utilización de plataformas virtuales e internet.

La percepción de satisfacción de los niveles de calidad educativa en la muestra de los estudiantes de la carrera de Redes y Telecomunicaciones del Instituto Superior Tecnológico "Luis A. Martínez" en diciembre del 2019, apuntaba a una deficiente utilización de las Tecnologías de la información y Comunicación debido a que no había la necesidad de utilizar dichas herramientas. 
Existen algunas herramientas que por diversas razones no se han utilizado académicamente en alguna asignatura del instituto, sin embargo en el presente trabajo se sugiere utilizarlas como recursos complementarios como es el caso de:

Wolfram Alpha: Es una biblioteca digital a modo de calculadora online, muy apta para modelado matemático y cálculos complejos relacionados a ingeniería, posee una alta capacidad para analizar gráficamente cualquier tipo de función.

\section{Desventajas:}

Se requiere internet activo para acceder a cualquiera de sus servicios.

Para utilizar todas las funciones hay que registrarse y ello acarrea costos.

Simbolab: es muy similar a Wolfram Alpha, se sugiere usarla en matemáticas para potenciar el análisis de funciones reales mediante sus gráficas, o para trabajar los contenidos estadísticos descriptivos.

Livewire: simulador electrónico desatendido y gratuito, ligero, se sugiere trabajar con esta herramienta en algunos módulos como: Algebra Booleana para comprobar funciones lógicas, en Electricidad y Magnetismo, para probar el funcionamiento de elementos electrónicos como la resistencia y el condensador.

\section{Conclusiones.}

- Se encontró una gran variedad de herramientas tecnológicas versátiles, económicas y vanguardistas que se pueden utilizar para el aprendizaje y enseñanza complementaria de temáticas importantes relacionadas con el perfil de la carrera de Redes y telecomunicaciones como es el caso de Simuladores online y software de simulación gratuitos, sin embargo el nivel de disponibilidad de tecnología y servicio de internet no es el esperado.

- Durante el trámite de la investigación se sugieren algunas herramientas TIC para complementar el aprendizaje y competencias importantes en la configuración y manipulación adecuada los equipos de redes y telecomunicaciones como Packet Tracer, Crocodile technology o Livewire, en beneficio de los estudiantes de Redes y Telecomunicaciones.

- El nivel de uso de medios tecnológicos mejoró considerablemente debido a la creciente necesidad de utilizar plataformas virtuales y clases por video conferencia en el 2020 como efecto del cambio de la modalidad de estudios obligatoria debido a la pandemia del covid -19, sin embargo se detectó una mejoría promedio del 30\% en la utilización de TIC en los estudiantes que fueron motivados durante el periodo de diciembre a abril 2020.

- Los niveles de satisfacción en los estudiantes de la carrera de Redes y Telecomunicaciones en cuanto aprendizaje significativo se incrementaron positivamente, de tal forma que la mayoría considera que herramientas Tic como los simuladores electrónicos online o software se simulación permanente son 
fundamentales para comprender de mejor manera los contenidos de la especialidad, más aún en la realidad actual e la educación superior.

\section{Referencias Bibliográficas}

Appsherramientasmultimediaonlineval. (08 de 04 de 2016). Aplicaciones de herramientas multimedia online. obtenido de appsherramientasmultimediaonlineval: https://appsherramientasmultimediaonlineval.wordpress.com/2016/04/08/aplicacion es-de-herramientas-multimedia-online/

aula1. (10 de 2020). Plataformas educativas ¿Qué son y para qué sirven? Obtenido de Plataformas educativas ¿Qué son y para qué sirven?: https://www.aula1.com/plataformas-educativas/

Biblioteca de la Universidad de Alcalá. (10 de 2020). Biblioteca de la Universidad de Alcalá. Obtenido de Fuentes de Información: http://www3.uah.es/bibliotecaformacion/BPOL/FUENTESDEINFORMACION/tip os_de_fuentes_de_informacin.html

Bojórquez Molina, J. A., López Aranda, L., Hernández Flores, M. E., \& Jiménez López, E. (2013). Utilización del alfa de Cronbach para validar la confiabilidad de. Eleventh LACCEI Latin American and Caribbean Conference for Engineering and Technology, 1-9.

Castellanos, M. (2015). ¿Son las TIC realmente, una herramienta valiosa para fomentar la calidad de la educación? Obtenido de UNESCO: http://unesdoc.unesco.org/images/0024/002449/244952S.pdf

CES. (30 de 06 de 2017). RPC-SO-23-No.459-2017. Rediseño Curricular de la Carrera de Tecnología Superior en Redes y Telecomunicaciones. Quito, Pichincha, Ecuador: CES.

Consejo de Educación Superior. (25 de 02 de 2019). RPC-SO-08-No.111-2019. REGLAMENTO DE RÉGIMEN ACADÉMICO. Quito, Pichincha, Ecuador: CES.

FisicaLab. (10 de 2020). Ley de Ohm. Obtenido de Ley de Ohm: https://www.fisicalab.com/apartado/ley-de-ohm

Gallardo, L., \& Buleje, J. (2010). Importancia de las TIC en la Educación. Investigación Educativa, 4.

Gallego, C. F. (2004). Cálculo del tamaño de la muestra. Matronas profesión, 5-13.

García-Valcárcel, A. (10 de 01 de 2001). La actitud de los futuros maestros hacia. Edutec 97. Obtenido de http//.www.ieev.uma.es/

George, D., \& Mallery. (2003). Alfa de Cronbach y consistencia interna de los ítems de un instrumento de medida. Revista de estudios Interdisciplinarios en Ciencias Sociales, 3-9.

Hofacker, A. (2008). Rapid lean construction - quality rating model. Manchester: s.n.

Koskela, L. (1992). Application of the new production philosophy to construction. Finland: VTT Building Technology.

LOES. (02 de 10 de 2010). Ley Orgánica de Educación Superior. Ley Orgánica de Educación Superior. Quito, Pichincha, Ecuador: Registro Oficial. 
ISSN: 2600-5859

Vol. 3, $\mathrm{N}^{\circ}$ 4, p. 146-163, octubre-diciembre, 2020

Lucas, M. (28 de 08 de 2009). Automatismos Mar del Plata. Obtenido de Livewire,simulador electrónico: $\quad$ https://www.automatismos-mdq.com.ar/blog/2009/08/livewiresimulador-electronico.html

Ruiz, J., Martínez, M., \& Sánchez, M. (2016). El impacto de las TICs en la calidad de la educación superior. Revista de Investigación en Ciencias Contables y Administrativas, 29.

Tello, E. (2011). Las tecnologías de la información y comunicaciones (TIC) y la brecha digital: su impacto en la sociedad de México. RUSC, 10. doi:DOI: https://doi.org/10.15517/eci.v1i1.33052

Thompson, \& Strickland. (2004). Concepto de las TICS. Obtenido de Concepto de las TICS: https://tecnologymao92.weebly.com/concepto-de-las-tics.html

\section{LCiencia}




\section{PARA CITAR EL ARTÍCULO INDEXADO.}

Tisalema Tasigchana, S. H., Borja Guevara, C. O., \& Godoy Arce, W. A. (2020). Herramientas TIC en la enseñanza online de competencias complementarias de redes y telecomunicaciones en los Institutos Superiores Tecnológicos de la provincia de Tungurahua. ConcienciaDigital, $\quad 3(4), \quad 146-163$. https://doi.org/10.33262/concienciadigital.v3i4.1448

\section{Ciencia LDigital}

El artículo que se publica es de exclusiva responsabilidad de los autores y no necesariamente reflejan el pensamiento de la Revista Conciencia Digital.

El artículo queda en propiedad de la revista y, por tanto, su publicación parcial y/o total en otro medio tiene que ser autorizado por el director de la Revista Conciencia Digital.

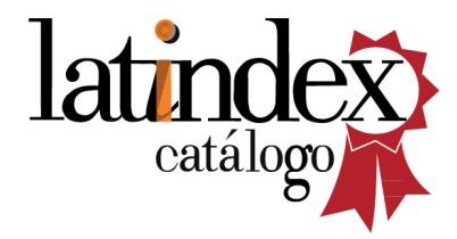

